MATHEMATICS OF COMPUTATION

Volume 67, Number 223, July 1998, Pages 1207-1224

S 0025-5718(98)00961-2

\title{
THE TRANSLATION PLANES OF ORDER 49 AND THEIR AUTOMORPHISM GROUPS
}

\author{
C. CHARNES AND U. DEMPWOLFF
}

\begin{abstract}
Using isomorphism invariants, we enumerate the translation planes
of order 49 and determine their automorphism groups.
\end{abstract}

\section{INTRODUCTION}

We describe the enumeration of the translation planes of order 49 and the computation of their automorphism groups. We follow the same pattern of classification used by the second author in [10] to handle the translation planes of order 27 , but we use slightly more refined methods.

The classification of the translation planes of order 49 was also obtained previously by R. Mathon and G. Royle [15]. However, these authors use quite different methods. In particular our solution to the isomorphism problem, a significant component of any enumeration of projective planes of a fixed order, is entirely different. We systematically use isomorphism invariants to solve this problem. This permits a different search strategy than that used in [15], resulting in a significant reduction in the computational effort. As a consequence, the complete enumeration of isomorphism classes of the spread sets corresponding to the translation planes of order 49 , can be repeated by anyone with standard computing resources.

The isomorphism invariants we use originate from an invariant of general (finite) projective planes which was proposed by J. H. Conway and investigated by C. Charnes in [3]; see [4]. To distinguish the isomorphism classes of translation planes, we use the fingerprint, the Leitzahl and Kennzahl, which are defined in [4] and [10], respectively. These invariants can be computed for the translation planes of order 49 without much overhead. Furthermore, our invariants can be used to determine the automorphism groups of the planes. Therefore, they are of independent interest, and we give in $\S 3$ a self-contained description of the invariants and of our algorithm for generating the spread sets.

We provide in $\S 7$ a detailed description of the automorphism groups of the translation planes of order 49. For each plane we give the following information: the order of the automorphism group; the order of the center; the order of the Fitting factor group; orders of the factors of the derived series; orders of the factors of the composition series; orders of the factors of the lower central series. Structural information of this kind is useful in the study of the geometrical properties of the planes and should suffice to identify each group. The orders of the groups were computed in two independent ways. First by U. Dempwolff, who determined the

Received by the editor July 3, 1995 and, in revised form, April 23, 1997.

1991 Mathematics Subject Classification. Primary 51E15, 68R05, 05B25.

(C)1998 American Mathematical Society 
elements of $G L_{4}(7)$ which leave invariant each spread set. The group orders were computed from these generators with a program written by U. Dempwolff. Independently, GAP [16] routines were used to obtain the aforementioned data from the generating matrices.

In [6] we announced the classification of the translation planes of order 49 whose automorphism groups contain involutory homologies. These planes were used as a check on the correctness of the complete enumeration - each involutory homology plane had to occur in the final list of planes. Finally, the number of isomorphism classes of translation planes of order 49 we obtain, 973 up to polarity, agrees with the enumeration in [15].

In $\S 5$ we identify the involutory homology planes and some other planes which have appeared in the literature.

\section{Definitions And nOtation}

We recall briefly the results and notation used in the enumeration of translation planes; details can be found in [10] and [13].

Let $W=V \oplus V, V=G F\left(p^{n}\right)$, be a $2 n$-dimensional vector space over $G F(p)$. A collection $\mathcal{S}=\left\{V_{\infty}, V_{0}, \ldots, V_{m}\right\}$ of mutually disjoint $n$-dimensional subspaces of $W$ is called a partial spread. If $m=p^{n}-1$, then $\mathcal{S}$ is a spread and describes a translation plane of order $p^{n}$. By choosing a basis in $W$ we can write $V_{\infty}=\{(0, v) \mid v \in V\}$, $V_{0}=\{(v, 0) \mid v \in V\}$ and $V_{i}=\left\{\left(v, v t_{i}\right) \mid v \in V\right\}$, where $t_{i} \in G=G L_{n}(p)$ and $t_{1}=1$. We call $S=\left\{t_{0}, t_{1}, \ldots, t_{m}\right\}$ a spread set with respect to the coordinate triple $(\infty, 0,1)$.

A basic property of spread sets is: $(*) \operatorname{det}\left(t_{i}-t_{j}\right) \neq 0$ for $0 \leq i<j \leq m$. Conversely, each set $S \subseteq G \cup\{0\}$ satisfying (*) defines a spread set. This description determines a spread set only up to conjugacy; see [10]. Replacing $\left(V_{\infty}, V_{0}, V_{1}\right)$ by some other triple $\left(V_{i}, V_{j}, V_{k}\right)$ defines a spread set $S^{\prime}$; this is a coordinatization of $S$ with respect to $(i, j, k)$.

The possible coordinatizations are obtained from each other by the successive application of the following operations:

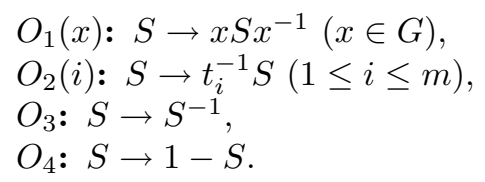

Definition 2.1. Two (partial) spread sets $S_{1}, S_{2}$ are said to be equivalent if and only if $S_{1}$ can be obtained from $S_{2}$ by a successive application of operations $\mathrm{O}_{1}(x), \ldots, \mathrm{O}_{4}$.

The problem of enumerating the isomorphism classes of translation planes of order $p^{n}$ reduces to the problem of determining a set of representatives of the equivalence classes induced by the above relation.

These equivalence operations can also be used to determine the automorphisms of the translation planes. For suppose that $t \in G L_{2 n}(p)$ is an automorphism of $\mathcal{S}$ and that $t$ maps $\left(V_{\infty}, V_{0}, V_{1}\right)$ to $\left(V_{i}, V_{j}, V_{k}\right)$. To determine $t$ amounts to finding a sequence of equivalence operations which take a spread set $S$ (with respect to $(\infty, 0,1))$ onto a spread set $S^{\prime}$ (with respect to $\left.(i, j, k)\right)$. To ease the computations, we also use the more crude weak equivalence, which is defined as: 
Definition 2.2. $S$ is weakly equivalent to $S^{\prime}$ if and only if $S$ or $S^{T}$ is equivalent to $S^{\prime}$.

\section{INVARIANTS}

We describe some invariants of (weak) equivalence. They are a decisive tool for the practical computation of the equivalence classes of spread sets. Let $\left\{x_{0}=\right.$ $\left.0, x_{1}, \ldots, x_{m}\right\}$ be a spread set.

Fingerprint [4], [10]. For $x \in G F(p)^{n \times n}$ set $[x]=\left(\frac{\operatorname{det} x}{p}\right.$, the Legendre symbol, if

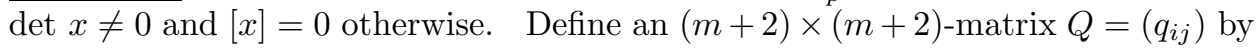

$$
\begin{gathered}
q_{i j}=\left|\sum_{k=0}^{m}\left[x_{i}-x_{k}\right]\left[x_{j}-x_{k}\right]+1\right|, \quad 0 \leq i, j \leq m ; \\
q_{i \infty}=q_{\infty i}=\left|\sum_{k=0}^{m}\left[x_{i}-x_{k}\right]\right|, \quad 0 \leq i \leq m, q_{\infty \infty}=m+1 .
\end{gathered}
$$

Then the multiset of entries of $Q=Q(S)$ is an invariant of equivalence.

Fingerprints were used in [4] to determine the canonical forms of the ovoids which correspond (by the Klein correspondence) to translation planes of order $p^{2}$; see also [7]. The automorphism groups of translation planes can be determined in this way; see [4] and [5].

Leitzahl [6].For $x \in G F(p)^{n \times n}$ define $\ll x \gg=1$ if $\operatorname{det} x=1$ and $\ll x \gg=0$ otherwise. Set

$$
\ell(S)=\sum_{0 \leq i<j \leq m} \sum_{\substack{k \neq i, j \\ k=0}}^{m} \ll\left(x_{i}-x_{k}\right)\left(x_{k}-x_{j}\right)^{-1} \gg .
$$

Then $\ell(S)$ is invariant with respect to all equivalence operations except possibly $O_{3}$. Thus, if $S_{1}$ and $S_{2}$ are two coordinatizations of a spread set with respect to $\left(a, b_{1}, c_{1}\right)$ and $\left(a, b_{2}, c_{2}\right)$, respectively, then $\ell\left(S_{1}\right)=\ell\left(S_{2}\right)$.

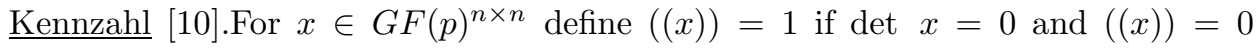
otherwise. Set

$$
k(S)=\sum_{1 \leq i<j \leq m}\left(\left(x_{i}+x_{j}\right)\right)
$$

Then $k(S)$ is invariant with respect to all equivalence operations except possibly $O_{4}$. Thus, if $S_{1}$ and $S_{2}$ are two coordinatizations of a spread set with respect to $\left(a_{1}, b_{1}, c_{1}\right)$ and $\left(a_{2}, b_{2}, c_{2}\right)$, respectively, then $k\left(S_{1}\right)=k\left(S_{2}\right)$ if $\left\{a_{1}, b_{1}\right\}=\left\{a_{2}, b_{2}\right\}$.

Finally, we denote by $c(S)$ the multiset of numbers $|S \cap C|$, where $C$ ranges over the conjugacy classes of $G$. Clearly, $c(S)$ is a conjugacy invariant.

Next, we give an algorithm which uses the above mentioned invariants to test the equivalence of two spread sets $S, S^{\prime}$. 


\section{Algorithm}

Step 1: If $Q(S) \neq Q\left(S^{\prime}\right)$, then stop. Otherwise:

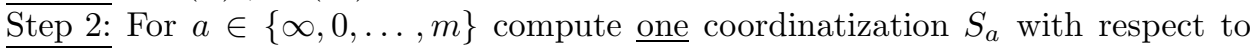
$\overline{(a, *, *)}$ (for an arbitrary admissible $*$ ). If always $\ell\left(S_{a}\right) \neq \ell\left(S^{\prime}\right)$, then stop. Otherwise:

Step 3: For $b \in\{\infty, 0, \ldots, m\} \backslash\{a\}$ compute one coordinatization $S_{a b}$ with respect to $(a, b, *)$. If always $k\left(S_{a b}\right) \neq k\left(S^{\prime}\right)$, then repeat Step 2 with $a:=a+1$ if possible. If not, then stop. Otherwise:

Step 4: For $c \in\{\infty, 0, \ldots, m\} \backslash\{a, b\}$ compute a coordinatization $S_{a b c}$ of $S$ with $\overline{\text { respect }}$ to $(a, b, c)$. If always $c\left(S_{a b c}\right) \neq c\left(S^{\prime}\right)$, then repeat Step 3 with $b:=b+1$ (or $b:=b+2$ if $a:=b+1)$ if possible. If not, then stop. Otherwise:

Step 5: Attempt to find a $x \in G$ such that $x S_{a b c} x^{-1}=S^{\prime}$.

Stop.

For more details regarding this algorithm see [10]. Precisely the same procedure (excluding Step 1), is used to determine the generators for the automorphism group of $\mathcal{S}$ (belonging to $S$ ). Again the details can be found in [10].

\section{THE SEARCH AND RESUlts}

The search for the representatives of the equivalence classes of spread sets of translation planes of order 49 routinely follows the search for the spread sets of the translation planes of order 27, described in [10]. In the order 49 case, we have used a similar classification of starter sets $S=\left\{t_{0}, t_{1}=1, \ldots, t_{m}\right\}$, where $6 \leq m \leq 12$. We choose $t_{2}, \ldots, t_{6}$ which fixes a particular subspace of $V$, say $t_{i}=\left(\begin{array}{c}* * \\ 0 *\end{array}\right)$.

If $k$ is the maximum number of scalar matrices in a coordinatization of $S$, we have to distinguish the six cases: $k=1, \ldots, 6$. In contrast to the search used by R. Mathon and G. Royle [15], we did not require a result like their Lemma 3.1. Instead we determined all completions in every case to gain extra control. (Distinguishing the isomorphism classes of completions is inexpensive with our methods.) Our final enumeration matches completely the enumeration described in [15].

There are precisely 973 representatives of spread sets up to weak equivalence. There are 374 spread sets which have the property that $S$ is inequivalent to $S^{T}$ (i.e., represent pairs of mutually polar planes). Thus, there are 1347 representatives of spread sets up to equivalence. For each representative spread set we have computed a set of matrix generators - elements of $G L_{4}(7)$, which leave the spread set invariant. From these generators we computed the data contained in $\S 7$.

4.1. Control. In such a large enumeration problem, it is important to first establish a control set. Therefore, we have independently determined the spread sets of the translation planes of order 49 which admit involutory homologies. There are precisely 154 such spread sets up to weak equivalence; see [6]. Heuristic reasons explained in [10] and [13] show that the identification of the 154 spread sets among the complete list of spread sets will provide a reasonable test of the correctness of the enumeration.

\section{IDENTIFICATION OF SOME KNOWN PLANES}

In this section we identify some planes of order 49 which have previously appeared in the literature with those occurring in our list. (For the notation see §7.)

As in [10], we have checked our list of spread sets for possible symplectic spreads. It turns out that only the Desarguesian spread $9 c u$ has this property. 
Planes with involutory homologies [6]:

\begin{tabular}{|llllllllll|}
\hline $0 a a$ & $0 a b$ & $0 a c$ & $0 a d$ & $0 a e$ & $0 a f$ & $0 a h$ & $0 a i$ & $0 a k$ & $0 a m$ \\
$0 a o$ & $0 a q$ & $0 a s$ & $0 a v$ & $0 a w$ & $0 a x$ & $0 a y$ & $0 b b$ & $0 b e$ & $0 b k$ \\
$0 b l$ & $0 b r$ & $0 b s *$ & $0 b x$ & $0 c c$ & $0 c g$ & $0 c h$ & $0 c k$ & $0 c l$ & $0 c m$ \\
$0 c s$ & $0 c z$ & $0 d f$ & $0 d k$ & $1 a b$ & $1 a c$ & $1 a f$ & $1 a m *$ & $1 a n$ & $1 a q$ \\
$1 b d$ & $1 b e$ & $1 b q$ & $1 b r$ & $1 c k *$ & $1 c m$ & $1 c s *$ & $1 c t$ & $1 d l$ & $1 d m$ \\
$2 b t$ & $2 b u$ & $2 c v$ & $2 d c$ & $2 d d *$ & $3 a f *$ & $3 a n$ & $3 a o$ & $3 a p$ & $3 a q$ \\
$3 a s$ & $3 d b$ & $3 d p$ & $4 a d$ & $4 a n$ & $4 b i$ & $4 b m$ & $4 c j *$ & $4 d b$ & $4 d s$ \\
$5 a f$ & $5 a n$ & $5 a w$ & $5 b q$ & $5 b r$ & $5 b s$ & $5 b t$ & $5 c s *$ & $5 c u$ & $6 a m$ \\
$6 a u$ & $6 c b$ & $6 c e *$ & $6 c s$ & $6 d j *$ & $6 d l$ & $6 d m$ & $7 a b$ & $7 a m *$ & $7 a o$ \\
$7 b e$ & $7 b i$ & $7 b s$ & $7 b z$ & $7 c s$ & $7 c y$ & $7 d d$ & $7 d f$ & $7 d p$ & $7 d q *$ \\
$7 d s *$ & $8 a g *$ & $8 a l$ & $8 a s$ & $8 a t$ & $8 b l$ & $8 c e$ & $8 c h$ & $8 c o$ & $8 c w$ \\
$8 c x$ & $8 d e$ & $8 d h$ & $8 d l$ & $8 d q$ & $9 a a$ & $9 a j$ & $9 a k$ & $9 a t$ & $9 a w$ \\
$9 a z$ & $9 b g$ & $9 b h$ & $9 b i$ & $9 b j$ & $9 b k$ & $9 b l$ & $9 b m$ & $9 b p$ & $9 b q *$ \\
$9 b r$ & $9 b t$ & $9 b v$ & $9 b w$ & $9 b y$ & $9 b z$ & $9 c a$ & $9 c c$ & $9 c d$ & $9 c e$ \\
$9 c g$ & $9 c h$ & $9 c j$ & $9 c k$ & $9 c l$ & $9 c m$ & $9 c n$ & $9 c o$ & $9 c p$ & $9 c q$ \\
$9 c r$ & $9 c s$ & $9 c t$ & $9 c u$ & & & & & & \\
\hline
\end{tabular}

Heimbeck planes with quaternion group of homologies [11]:

\begin{tabular}{|l|l|l|r|}
\hline Name & Name in [11] & Order & Orbits on $l_{\infty}$ \\
\hline $9 c q$ & I & 13824 & 248 \\
$0 d f$ & II & 6912 & 248 \\
$9 c t$ & III & 11520 & 1040 \\
$3 a n$ & IV & 9216 & 248 \\
$0 a f$ & V & 2304 & 21632 \\
$0 a c$ & VI & 3072 & 21632 \\
$4 a d$ & VII & 768 & 2161616 \\
$0 a d$ & VIII & 2304 & 21632 \\
$1 b e$ & IX & 1536 & 21632 \\
$9 c p$ & X & 576 & 1148 \\
\hline
\end{tabular}

Heimbeck plane with a shear [12]: 8dl, 2016, 1742.

Non-Desarguesian planes with nonsolvable group:

\begin{tabular}{|l|l|l|r|}
\hline Name & Name in the literature & Order & Orbits on $l_{\infty}$ \\
\hline $0 a b$ & Hall & 32256 & 842 \\
$0 a u$ & Mason & 4320 & 2030 \\
$0 b a$ & $S_{5}$-Type [7] & 1440 & 1040 \\
$9 b n$ & Mason & 4320 & 2030 \\
$9 c s$ & $S_{5}$-Type [7] (also Korchmaros) & 2880 & 2030 \\
$9 c t$ & Mason-Ostrom & 11520 & 1040 \\
\hline
\end{tabular}




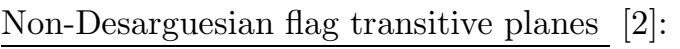

\begin{tabular}{|l|l|c|c|}
\hline Name & Name in $[2]$ & Order & Orbits on $l_{\infty}$ \\
\hline $0 a n *$ & {$\left[L_{1}\right] \cup\left[L_{1}^{\prime}\right],\left[L_{1}\right] \cup\left[L_{1}^{\prime \prime}\right]$} & 600 & 50 \\
$1 d r$ & {$\left[L_{3}\right] \cup\left[L_{3}^{\prime}\right]$} & 600 & 50 \\
\hline
\end{tabular}

Planes as projections of 8-dimensional ovoids: Certain translation planes of order 49 arise (by the Klein correspondence) from 6 -dimensional ovoids which are 'sections' of ovoids in $\Omega^{+}(8,7)$. There are two ovoids in $\Omega^{+}(8,7)$ which are invariant under the Weyl groups $W\left(D_{7}\right)$ [17], and $W\left(E_{7}\right)$ [8]. These give 16 isomorphism classes of translation planes which are indexed by the orbits of $W\left(D_{7}\right)$ and $W\left(E_{7}\right)$ acting on the isotropic vectors of $\Omega^{+}(8,7)$; see [8] and [3]. We identify these planes below. An entry in the 'ovoid' column indicates that the plane corresponds to a section of the listed 8-dimensional ovoid.

\begin{tabular}{|lll|lll|}
\hline Name & Ovoid & Orbits on $l_{\infty}$ & Name & Ovoid & Orbits on $l_{\infty}$ \\
\hline Oad & $D_{7} E_{7}$ & 21632 & $0 c a$ & $D_{7}$ & 681224 \\
0ae & $D_{7} E_{7}$ & 6836 & $1 a q$ & $D_{7} D_{7} D_{7}$ & 461624 \\
0ag & $D_{7} D_{7} E_{7}$ & 28121216 & $6 c s$ & $D_{7} D_{7}$ & 28121216 \\
$0 a k$ & $D_{7}$ & 21236 & $9 b n$ & $D_{7} D_{7} E_{7}$ & 2030 \\
$0 a u$ & $E_{7} E_{7}$ & 2030 & $9 c n$ & $D_{7} E_{7}$ & 81824 \\
$0 b a$ & $D_{7} D_{7} E_{7}$ & 1040 & $9 c q$ & $D_{7} E_{7}$ & 248 \\
$0 b e$ & $D_{7} D_{7} E_{7}$ & 33836 & $9 c s$ & $D_{7} E_{7}$ & 2030 \\
$0 b l$ & $D_{7}$ & 61232 & $9 c t$ & $D_{7} E_{7}$ & 1040 \\
\hline
\end{tabular}

\section{The Structure of the AUtOMORPhism Groups}

To describe the structure of the automorphism groups of the 1347 translation planes of order 49 , it suffices to consider only the 973 representatives up to polarity. Since a dual spread has the same abstract group in its contragradient representation, the two groups have the same orders.

For each representative spread set we calculate the following: the order of the automorphism group; the order of the center; the order of the Fitting factor group; orders of the factors of the derived series; orders of the factors of a composition series; orders of the factors of the lower central series. We also indicate which spreads are self-polar ( $S$ isomorphic to $S^{T}$ ). This data was obtained with GAP [16] with the help of the generators obtained by U. Dempwolff. The GAP routines were prepared mechanically using AWK [1] from the generators to prevent errors from creeping in.

6.1. Non-Abelian composition factors. Tables I - X, given in $\S 7$, show that the automorphism groups of the non-Desarguesian planes of order 49 have only the following non-Abelian composition factors: $P S L_{2}(5), P S L_{2}(7)$ and $P S L_{2}(9)$. Here, $P S L_{2}(7)$ gives the Hall plane, see [9], while $P S L_{2}(9)$ is of a Mason type, see [7]. Planes with $P S L_{2}(5)$ relate to the ones we considered in [7]. By the automorphism group, we mean the subgroup of $G L_{4}(7)$ generated by the spread stabilizer and the kernel homologies. 


\section{THE TABLES}

We present the automorphism groups of the translation planes of order 49 as follows. The 973 groups are split into 10 tables: Table I - X. The first 9 tables account for 900 groups, split into blocks of 100, the last table contains 73 groups. Each table contains the following information. The first five columns contain respectively: the name of the group - a name with an asterisk indicates a polar pair; the order of group; the order of the center; the order of the derived subgroup; the order of the Fitting factor group. The last three columns contain respectively: orders of the factor groups of the composition series; orders of the factors of the derived subgroup series; orders of the factors of the lower central series.

We omit a group from the tables if it is the group of central homologies (of order 6 ) and the corresponding plane is self-polar. For example, in Table I, 1at is omitted. (There are 246 such planes up to polarity.) In case that $G=Z(G)$ has order 6 (example $1 d f$ ), we give no composition series and no derived series. If $G=Z(G)$ has order greater than 6 (example $0 a s$ ), we omit the derived series.

7.1. The spread sets. The complete list of spread sets of the translation planes of order 49 is available at the following ftp site:

$$
\text { ftp://www.mathematik.uni-kl.de/pub/Math/Algebra/49_planes }
$$

Other information regarding these planes can also be found at this site.

TABLE I. 0aa-0dv.

\begin{tabular}{|c|c|c|c|c|c|c|c|}
\hline $0 a a$ & 960 & 6 & 160 & 10 & {$[3,2,5,2,2,2,2,2]$} & {$[6,5,16,2]$} & [6] \\
\hline $0 a b$ & 32256 & 6 & 1344 & 336 & {$[2,3,2,168,2,2,2,2]$} & {$[24,4]$} & {$[24,2,2]$} \\
\hline $0 a c$ & 3072 & 6 & 128 & 1 & {$[2,3,2,2,2,2,2,2,2,2,2]$} & {$[24,8,16]$} & {$[24,4,2,2,2,2,2]$} \\
\hline Oad & 2304 & 6 & 192 & 6 & {$[2,3,2,3,2,2,2,2,2,2]$} & {$[12,3,16,4]$} & [12] \\
\hline oae & 1728 & 6 & 72 & 12 & {$[2,3,2,3,2,2,2,3,2]$} & {$[24,9,4,2]$} & [24] \\
\hline oaf & 2304 & 6 & 192 & 6 & {$[2,3,2,3,2,2,2,2,2,2]$} & {$[12,3,16,4]$} & {$[12]$} \\
\hline $0 a g$ & 288 & 6 & 24 & 6 & {$[3,2,2,3,2,2,2]$} & {$[12,3,4,2]$} & {$[12]$} \\
\hline $\mathrm{Oah}$ & 288 & 12 & 24 & 6 & {$[2,3,2,3,2,2,2]$} & {$[12,3,4,2]$} & [12] \\
\hline $0 a i$ & 192 & 6 & 8 & 1 & {$[2,2,2,2,3,2,2]$} & {$[24,8]$} & {$[24,4,2]$} \\
\hline $0 a j$ & 432 & 6 & 36 & 8 & {$[2,3,2,2,2,3,3]$} & {$[12,4,9]$} & {$[12,2,2]$} \\
\hline $0 a k$ & 864 & 6 & 36 & 8 & {$[2,2,3,2,2,3,3,2]$} & {$[24,4,9]$} & {$[24,2,2]$} \\
\hline Oal & 96 & 6 & 8 & 1 & {$[2,2,2,3,2,2]$} & {$[12,8]$} & {$[12,2,2,2]$} \\
\hline Oam & 1536 & 6 & 32 & 1 & {$[2,2,2,2,2,3,2,2,2,2]$} & {$[48,32]$} & {$[48,4,4,2]$} \\
\hline oan* & 600 & 6 & 25 & 4 & {$[2,3,2,2,5,5]$} & {$[24,25]$} & [24] \\
\hline oao & 192 & 6 & 8 & 1 & {$[2,2,3,2,2,2,2]$} & {$[24,8]$} & {$[24,4,2]$} \\
\hline $0 a p$ & 288 & 6 & 24 & 2 & {$[2,3,3,2,2,2,2]$} & {$[12,24]$} & {$[12,2,2,2]$} \\
\hline $0 a q$ & 192 & 6 & 4 & 1 & {$[2,2,2,3,2,2,2]$} & {$[48,4]$} & {$[48,2,2]$} \\
\hline oar & 48 & 6 & 4 & 1 & {$[2,2,3,2,2]$} & {$[12,4]$} & {$[12,2,2]$} \\
\hline Oas & 24 & 24 & 1 & 1 & {$[2,2,3,2]$} & [] $]$ & {$[24]$} \\
\hline oat & 288 & 6 & 24 & 2 & {$[2,3,3,2,2,2,2]$} & {$[12,24]$} & {$[12,2,2,2]$} \\
\hline oau & 4320 & 6 & 720 & 720 & {$[2,360,3,2]$} & [6] & [6] \\
\hline oav & 768 & 6 & 32 & 1 & {$[2,2,2,3,2,2,2,2,2]$} & {$[24,32]$} & {$[24,4,4,2]$} \\
\hline Oaw & 288 & 12 & 24 & 6 & {$[2,3,2,3,2,2,2]$} & {$[12,3,4,2]$} & $[12], 1,2]$ \\
\hline oax & 1536 & 6 & 64 & 1 & {$[2,2,3,2,2,2,2,2,2,2]$} & {$[24,64]$} & {$[24,2,2,2,2,2,2]$} \\
\hline Oay & 384 & 6 & 16 & 1 & {$[2,2,2,3,2,2,2,2]$} & {$[24,16]$} & {$[24,2,2,2,2]$} \\
\hline $0 a z$ & 72 & 6 & 6 & 2 & {$[2,3,2,2,3]$} & {$[12,6]$} & {$[12,2]$} \\
\hline $\mathrm{Oba}$ & 1440 & 6 & 120 & 120 & {$[2,60,2,2,3]$} & [12] & [12] \\
\hline $\mathrm{Obb}$ & 864 & 6 & 36 & 8 & {$[2,2,3,2,2,2,3,3]$} & {$[24,4,9]$} & {$[24,2,2]$} \\
\hline $0 b c$ & 48 & 12 & 2 & 1 & {$[2,2,3,2,2]$} & {$[24,2]$} & {$[24,2]$} \\
\hline obd & 72 & 6 & 6 & 2 & {$[2,3,2,3,2]$} & {$[12,6]$} & {$[12,2]$} \\
\hline obe & 864 & 6 & 72 & 12 & {$[3,2,3,2,3,2,2,2]$} & {$[12,9,4,2]$} & [12] \\
\hline$o b f$ & 48 & 6 & 4 & 1 & {$[2,3,2,2,2]$} & {$[12,4]$} & {$[12,2,2]$} \\
\hline obg & 576 & 6 & 24 & 2 & {$[2,2,3,2,3,2,2,2]$} & {$[24,24]$} & {$[24,2,2,2]$} \\
\hline $\mathrm{obh}$ & 36 & 6 & 3 & 2 & {$[2,3,3,2]$} & {$[12,3]$} & {$[12]$} \\
\hline $\mathrm{Obi}$ & 48 & 6 & 4 & 1 & {$[2,3,2,2,2]$} & {$[12,4]$} & {$[12,2,2]$} \\
\hline $\mathrm{Obj}$ & 48 & 12 & 2 & 1 & {$[2,3,2,2,2]$} & {$[24,2]$} & {$[24,2]$} \\
\hline $\mathrm{obk}$ & 192 & 6 & 8 & 1 & {$[2,2,3,2,2,2,2]$} & {$[24,8]$} & {$[24,4,2]$} \\
\hline $\mathrm{obl}$ & 1152 & 6 & 96 & 6 & {$[2,3,2,2,2,3,2,2,2]$} & {$[12,12,4,2]$} & {$[12,2,2]$} \\
\hline $\mathrm{Obm}$ & 96 & 12 & 4 & 1 & {$[2,2,3,2,2,2]$} & {$[24,4]$} & {$[24,2,2]$} \\
\hline $0 b n$ & 192 & 6 & 8 & 1 & {$[2,2,3,2,2,2,2]$} & {$[24,8]$} & {$[24,2,2,2]$} \\
\hline obo & 12 & 12 & 1 & 1 & {$[3,2,2]$} & [] & {$[12]$} \\
\hline $0 b p$ & 96 & 12 & 4 & 1 & {$[2,2,3,2,2,2]$} & {$[24,4]$} & {$[24,2,2]$} \\
\hline $0 b q$ & 2016 & 6 & 168 & 12 & {$[3,2,2,7,3,2,2,2]$} & {$[12,21,4,2]$} & {$[12]$} \\
\hline $\mathrm{Obr}$ & 96 & 12 & 4 & 1 & {$[2,2,3,2,2,2]$} & {$[24,4]$} & {$[24,2,2]$} \\
\hline $0 b s *$ & 96 & 6 & 4 & 1 & {$[2,2,3,2,2,2]$} & {$[24,4]$} & {$[24,2,2]$} \\
\hline $0 b t$ & 96 & 6 & 4 & 1 & {$[2,2,3,2,2,2]$} & {$[24,4]$} & {$[24,2,2]$} \\
\hline obu & 96 & 12 & 4 & 1 & {$[2,2,3,2,2,2]$} & {$[24,4]$} & {$[24,2,2]$} \\
\hline $0 b v$ & 24 & 6 & 2 & 1 & {$[2,3,2,2]$} & {$[12,2]$} & {$[12,2]$} \\
\hline obw & 72 & 12 & 3 & 2 & {$[2,3,2,2,3]$} & {$[24,3]$} & {$[24]$} \\
\hline $0 b x$ & 576 & 6 & 48 & 6 & {$[2,3,2,2,3,2,2,2]$} & {$[12,6,4,2]$} & {$[12,2]$} \\
\hline oby & 48 & 6 & 4 & 1 & {$[2,2,3,2,2]$} & {$[12,4]$} & {$[12,2,2]$} \\
\hline $0 b z *$ & 288 & 6 & 12 & 2 & {$[2,2,3,3,2,2,2]$} & {$[24,12]$} & {$[24,2,2]$} \\
\hline
\end{tabular}


TABle I. (continued).
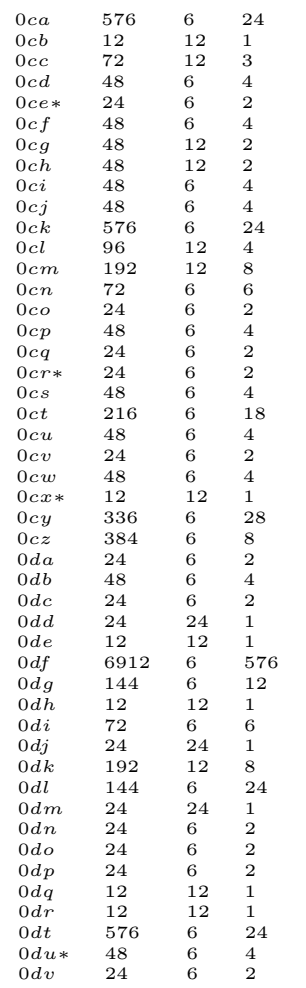

$[2,2,3,2,2,2,2,3]$
$[2,3,2]$
$[2,3,2,2,3]$
$[2,2,2,3,2]$
$[3,2,2,2]$
$[2,3,2,2,2]$
$[2,2,2,3,2]$
$[2,2,3,2,2]$
$[2,2,2,3,2]$
$[2,3,2,2,2]$
$[2,2,3,2,3,2,2,2]$
$[2,3,2,2,2,2]$
$[2,3,2,2,2,2]$
$[3,2,2,2,3]$
$[2,2,3,2]$
$[2,3,2,2,2]$
$[2,3,2,2]$
$[2,3,2,2]$
$[2,3,2,2,2]$
$[3,2,3,2,2,3]$
$[2,3,2,2,2]$
$[3,2,2,2]$
$[2,2,3,2,2]$
$[2,3,2]$
$[2,3,2,2,2,7]$
$[2,2,2,3,2,2,2,2]$
$[2,2,3,2]$
$[2,2,3,2,2]$
$[2,2,3,2]$
$[3,2,2,2]$
$[2,3,2]$
$[2,3,2,3,3,2,2,2,2,2,2]$
$[3,2,3,2,2,2]$
$[3,2,2]$
$[2,3,2,2,3]$
$[2,3,2,2]$
$[2,2,2,2,3,2,2]$
$[2,3,2,2,3,2]$
$[2,2,3,2]$
$[2,2,3,2]$
$[2,3,2,2]$
$[2,3,2,2]$
$[3,2,2]$
$[2,3,2]$
$[2,2,2,2,3,3,2,2]$
$[2,3,2,2,2]$
$[2,3,2,2]$

$\begin{array}{ll}{[24,24]} & {[24,2,2,2]} \\ {[]} & {[12]} \\ {[24,3]} & {[24]} \\ {[12,4]} & {[12,2,2]} \\ {[12,2]} & {[12,2]} \\ {[12,4]} & {[12,2,2]} \\ {[24,2]} & {[24,2]} \\ {[24,2]} & {[24,2]} \\ {[12,4]} & {[12,2,2]} \\ {[12,4]} & {[12,2,2]} \\ {[24,3,4,2]} & {[24]} \\ {[24,4]} & {[24,2,2]} \\ {[24,8]} & {[24,4,2]} \\ {[12,6]} & {[12,2]} \\ {[12,2]} & {[12,2]} \\ {[12,4]} & {[12,2,2]} \\ {[12,2]} & {[12,2]} \\ {[12,2]} & {[12,2]} \\ {[12,4]} & {[12,2,2]} \\ {[12,18]} & {[12,2]} \\ {[12,4]} & {[12,2,2]} \\ {[12,2]} & {[12,2]} \\ {[12,4]} & {[12,2,2]} \\ {[]} & {[12]} \\ {[12,28]} & {[12,2,2]} \\ {[48,8]} & {[48,2,2,2]} \\ {[12,2]} & {[12,2]} \\ {[12,4]} & {[12,2,2]} \\ {[12,2]} & {[12,2]} \\ {[]} & {[24]} \\ {[]} & {[12]} \\ {[12,9,16,4]} & {[12]} \\ {[12,12]} & {[12,2,2]} \\ {[]} & {[12]} \\ {[12,6]} & {[12,2]} \\ {[]} & {[24]} \\ {[24,8]} & {[24,4,2]} \\ {[6,3,4,2]} & {[6]} \\ {[]} & {[24]} \\ {[12,2]} & {[12,2]} \\ {[12,2]} & {[12,2]} \\ {[12,2]} & {[12,2]} \\ {[]} & {[12]} \\ {[]} & {[12]} \\ {[24,24]} & {[24,2,2,2]} \\ {[12,4]} & {[12,2,2]} \\ {[12,2]} & {[12,2]} \\ & \end{array}$


TABLE II. $1 \mathrm{aa}-1 \mathrm{dv}$.

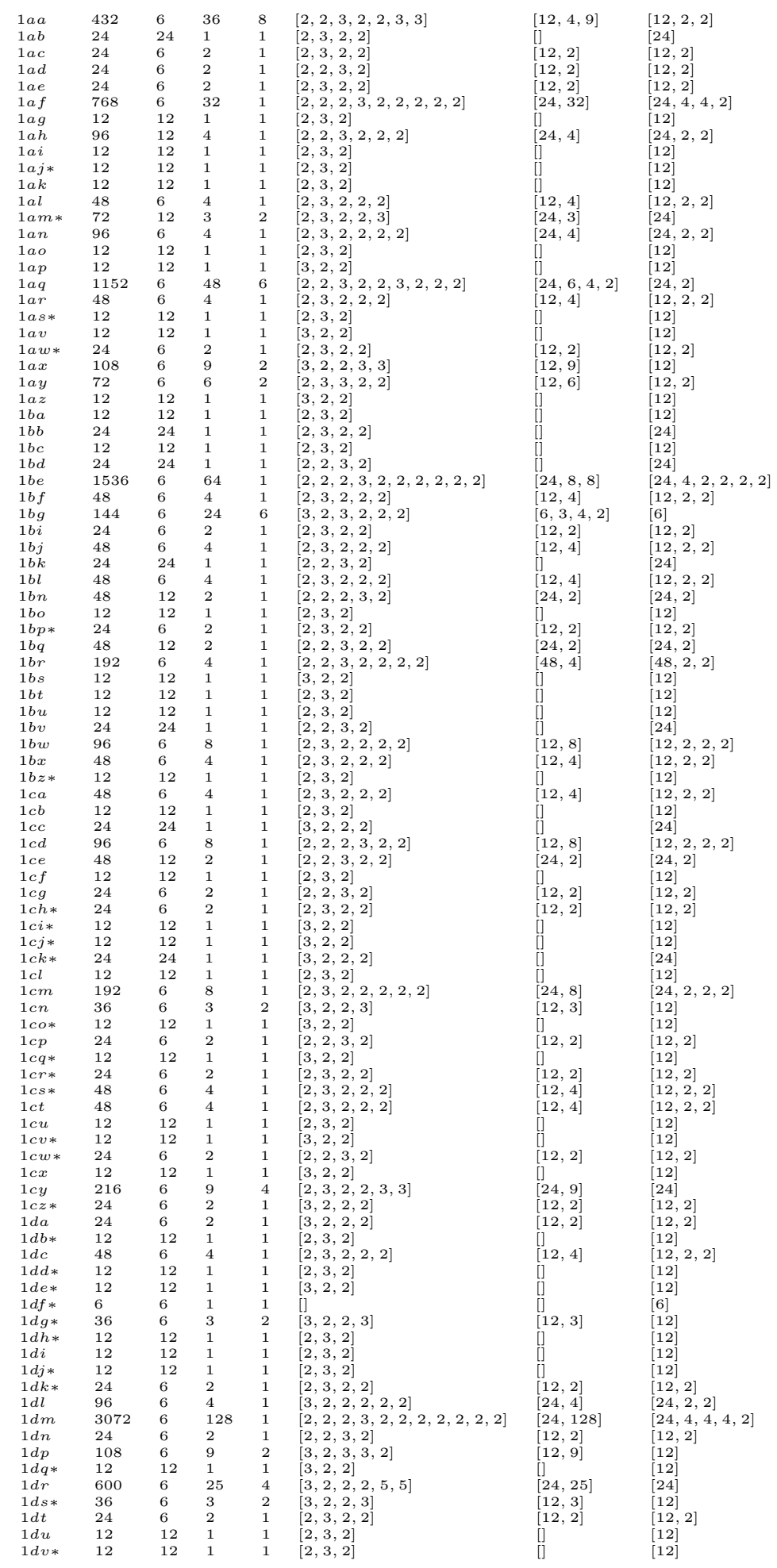


TABle III. 2aa-2dv.

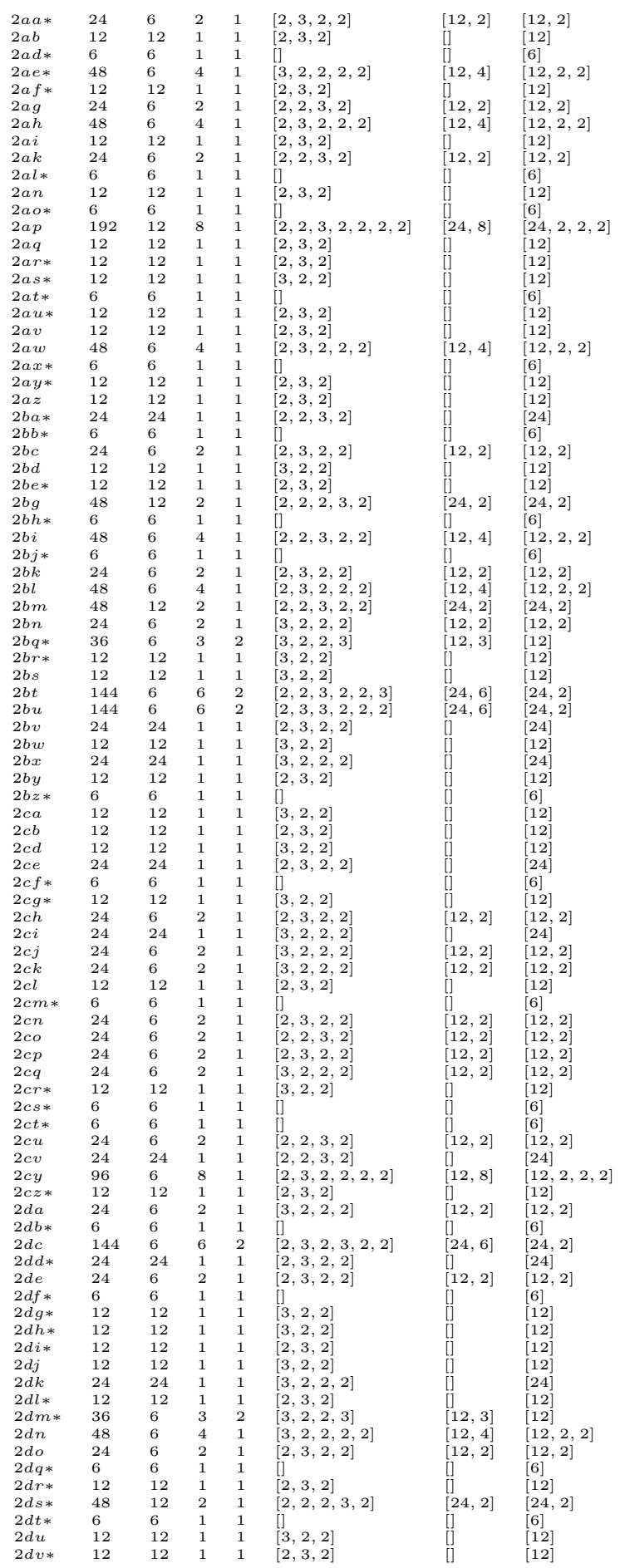


TABLE IV. 3aa-3dv.

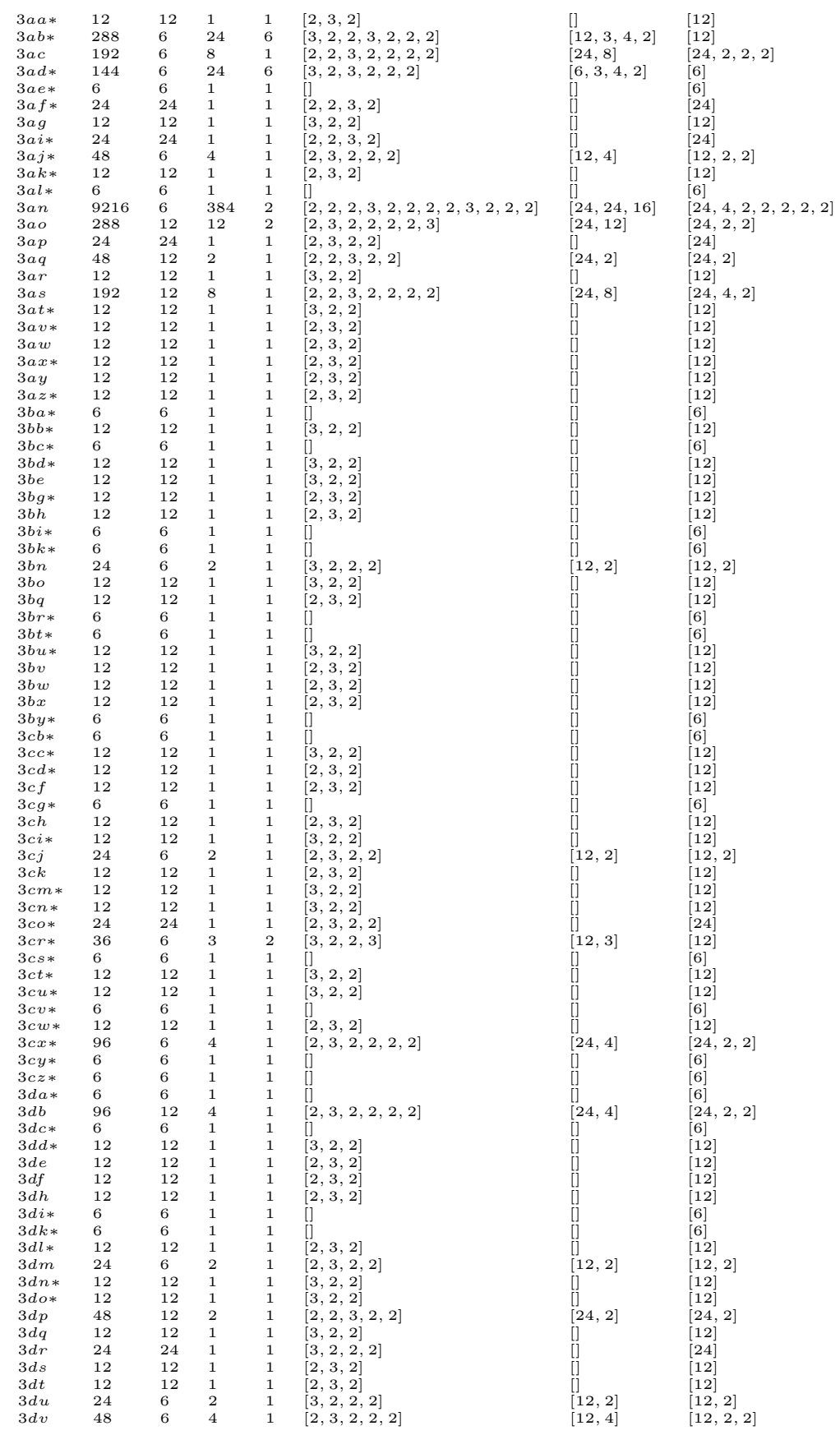


TABle V. 4aa-4dv.

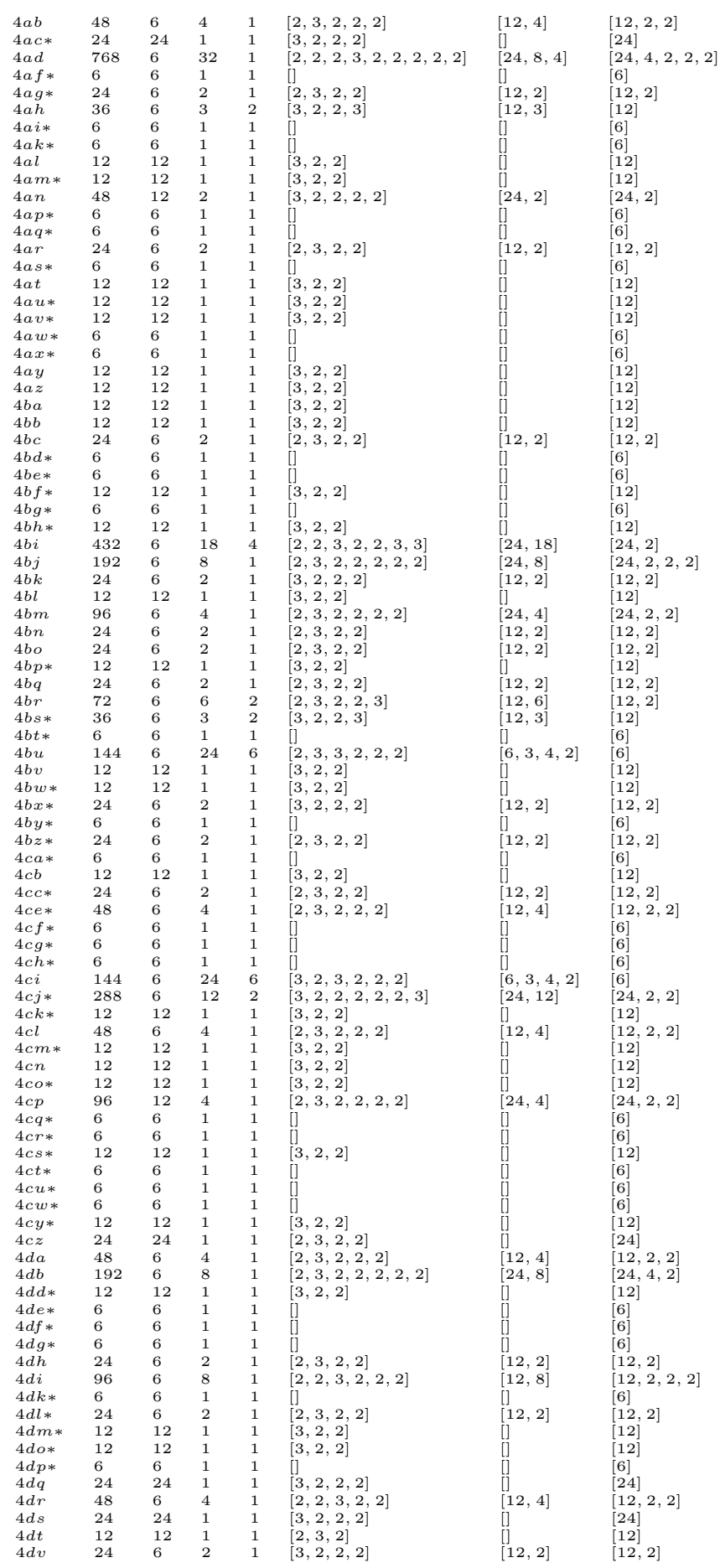


TABLE VI. 5aa- $-5 \mathrm{dv}$.

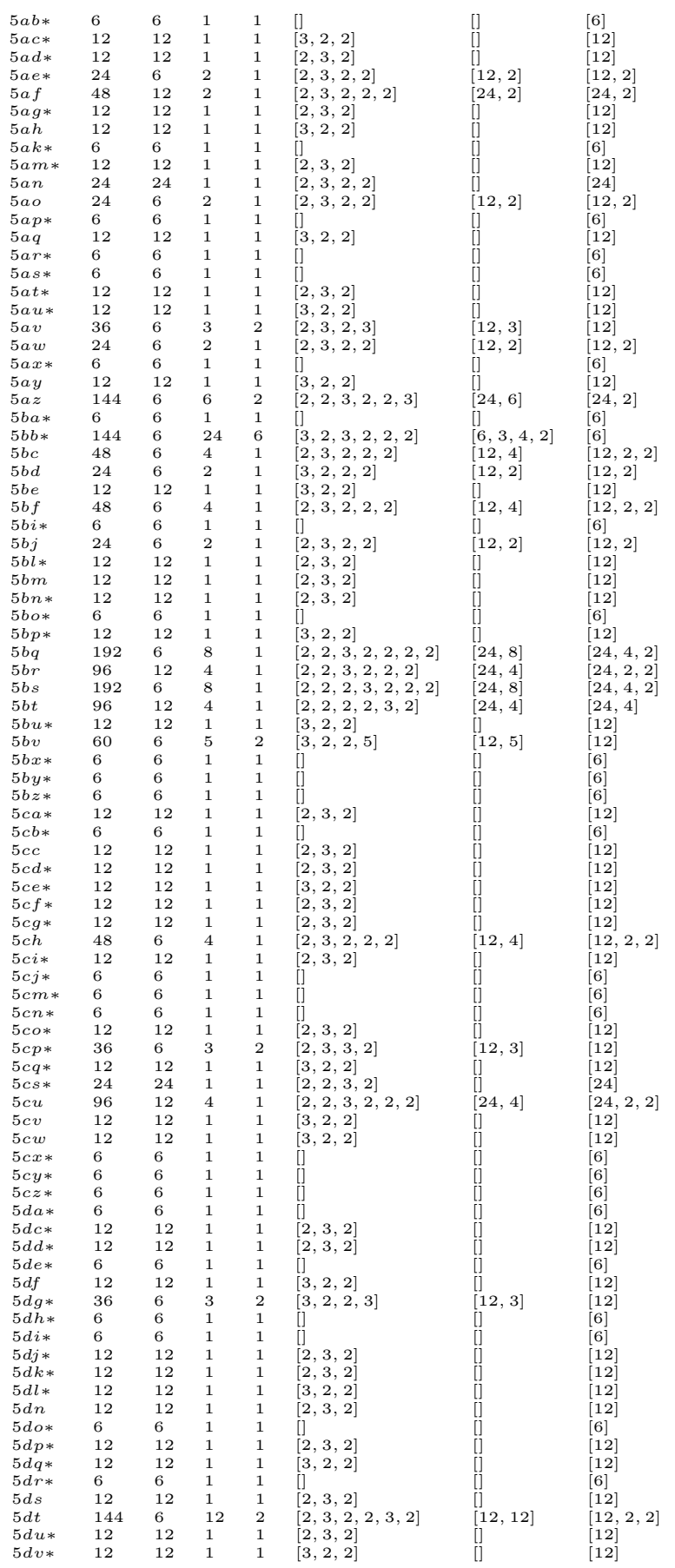


TABle VII. 6aa-6dv.

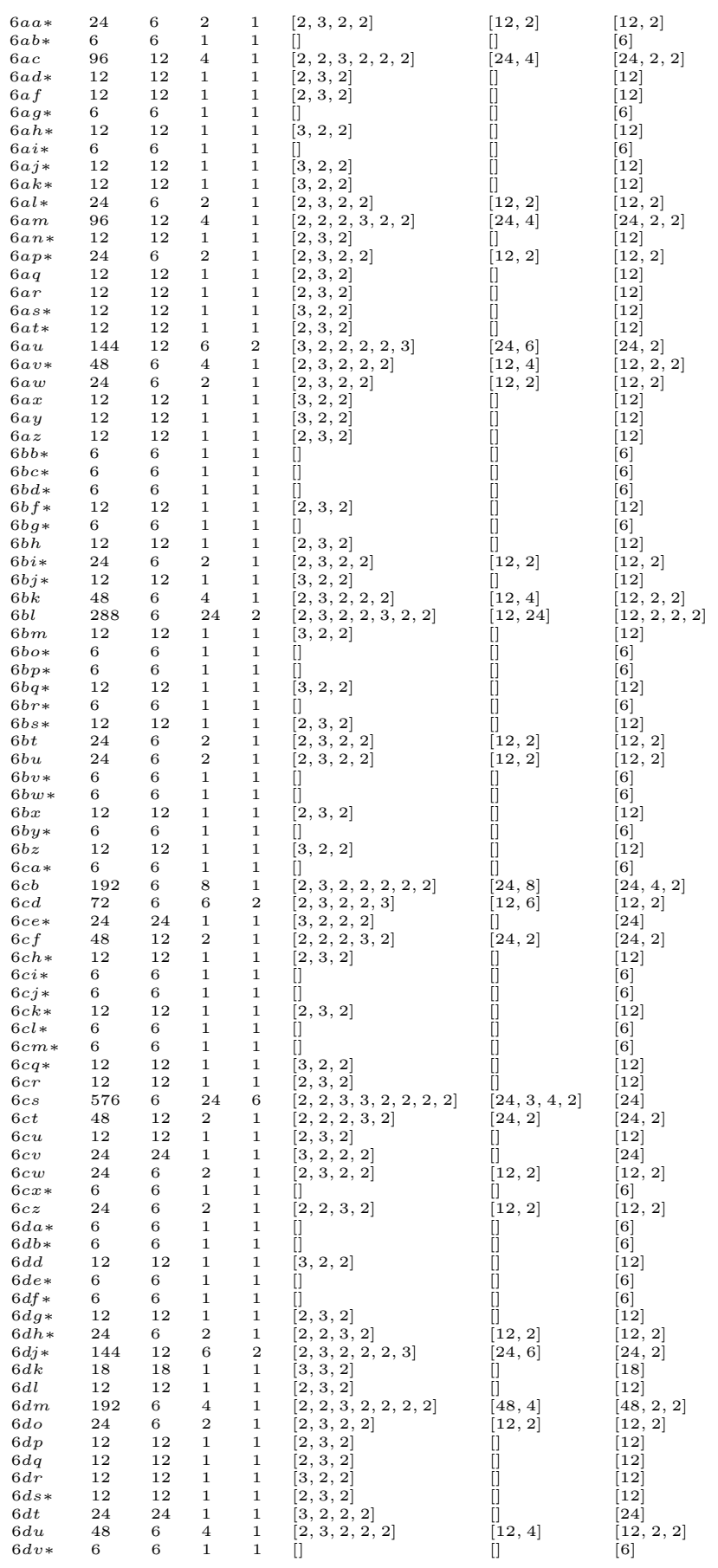


TABLE VIII. 7aa-7dv.

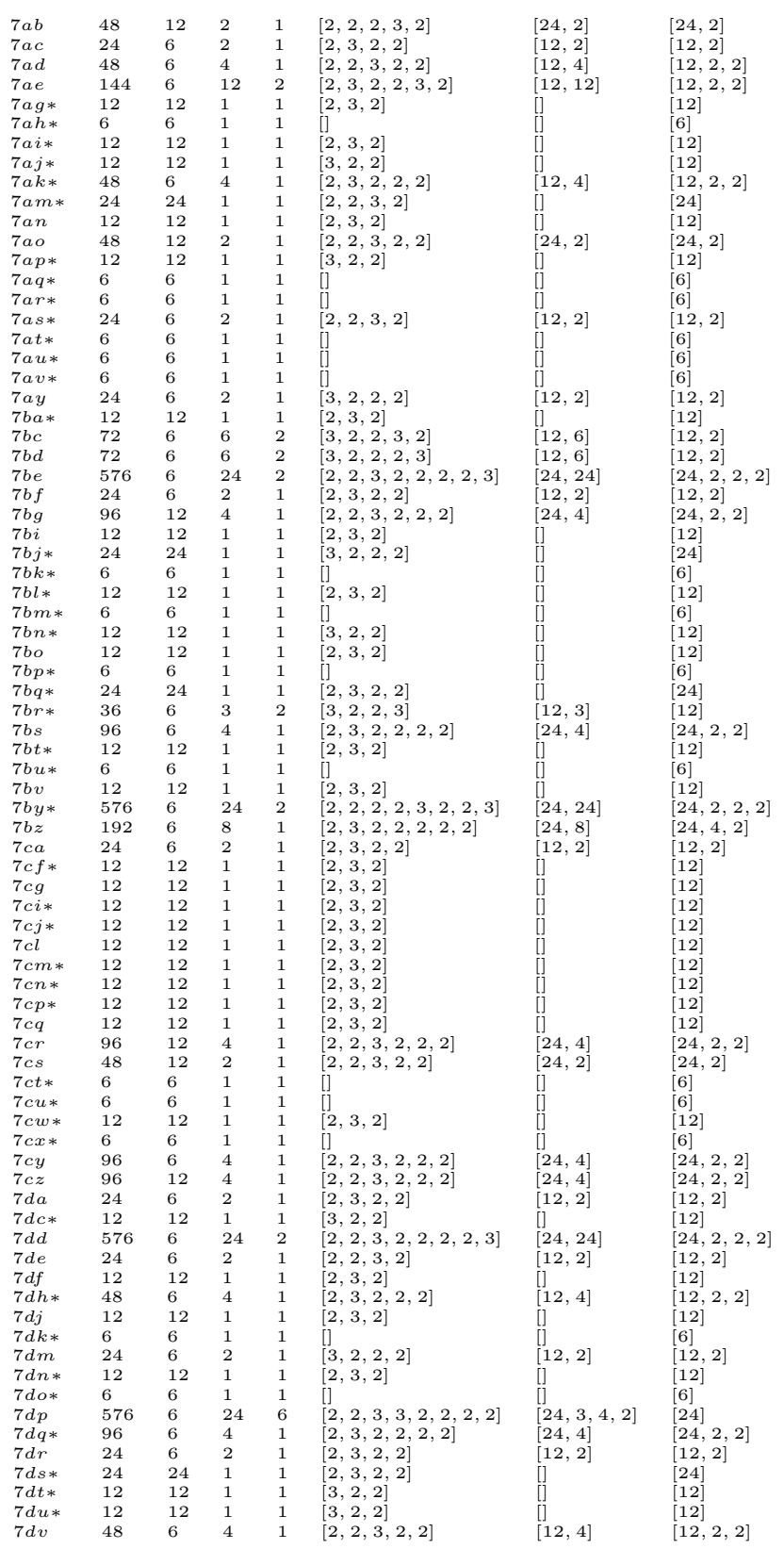


TABle IX. 8aa-8dv.

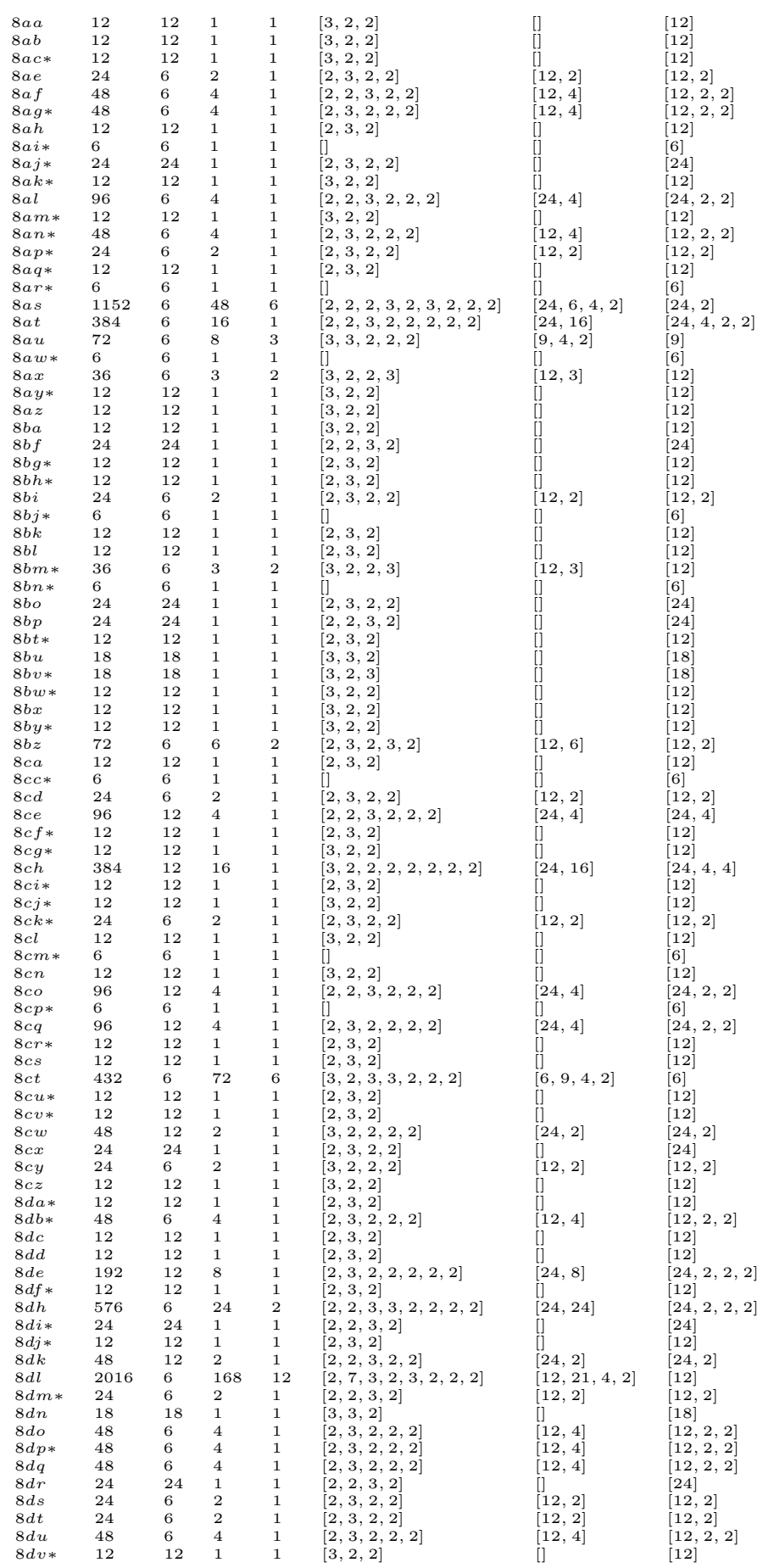


TABle X. 9aa-9cu.

\begin{tabular}{|c|c|c|c|c|c|c|c|}
\hline $9 a a$ & 48 & 6 & 4 & 1 & {$[2,3,2,2,2]$} & {$[12,4]$} & {$[12,2,2]$} \\
\hline $9 a b$ & 72 & 6 & 8 & 3 & {$[3,3,2,2,2]$} & {$[9,4,2]$} & \\
\hline $9 a c *$ & 12 & 12 & 1 & 1 & {$[3,2,2]$} & [] & {$[12]$} \\
\hline $9 a d *$ & 12 & 12 & 1 & 1 & {$[2,3,2]$} & [] & {$[12]$} \\
\hline $9 a f$ & 12 & 12 & 1 & 1 & {$[2,3,2]$} & [] & {$[12]$} \\
\hline $9 a g *$ & 12 & 12 & 1 & 1 & {$[2,3,2]$} & {$[1]$} & {$[12]$} \\
\hline $9 a h$ & 96 & 6 & 8 & 1 & {$[2,3,2,2,2,2]$} & {$[12,8]$} & {$[12,2,2,2]$} \\
\hline $9 a i *$ & 48 & 6 & 4 & 1 & {$[2,2,3,2,2]$} & {$[12,4]$} & {$[12,2,2]$} \\
\hline $9 a j$ & 240 & 6 & 10 & 4 & {$[2,3,2,2,2,5]$} & {$[24,10]$} & {$[24,2]$} \\
\hline $9 a k$ & 48 & 12 & 2 & 1 & {$[3,2,2,2,2]$} & {$[24,2]$} & {$[24,2]$} \\
\hline $9 a l$ & 12 & 12 & 1 & 1 & {$[3,2,2]$} & [] & {$[12]$} \\
\hline $9 a m$ & 12 & 12 & 1 & 1 & {$[2,3,2]$} & [] & {$[12]$} \\
\hline 9an* & 12 & 12 & 1 & 1 & {$[2,3,2]$} & [j] & {$[12]$} \\
\hline $9 a o$ & 48 & 6 & 4 & 1 & {$[3,2,2,2,2]$} & {$[12,4]$} & {$[12,2,2]$} \\
\hline $9 a p$ & 144 & 6 & 24 & 6 & {$[3,2,3,2,2,2]$} & {$[6,3,4,2]$} & {$[6]$} \\
\hline $9 a q *$ & 24 & 6 & 2 & 1 & {$[2,2,3,2]$} & {$[12,2]$} & {$[12,2]$} \\
\hline $9 a r *$ & 36 & 6 & 3 & 2 & {$[3,2,3,2]$} & {$[12,3]$} & {$[12]$} \\
\hline $9 a s *$ & 6 & 6 & 1 & 1 & [] & & {$[6]$} \\
\hline $9 a t$ & 192 & 6 & 8 & 1 & {$[3,2,2,2,2,2,2]$} & {$[24,8]$} & {$[24,4,2]$} \\
\hline $9 a v$ & 72 & 12 & 3 & 2 & {$[2,2,3,3,2]$} & {$[24,3]$} & $[24], 2]$ \\
\hline $9 a w$ & 216 & 12 & 9 & 2 & {$[2,3,2,2,3,3]$} & {$[24,9]$} & {$[24]$} \\
\hline $9 a x *$ & 36 & 6 & 3 & 2 & {$[2,3,3,2]$} & {$[12,3]$} & {$[12]$} \\
\hline $9 a y$ & 36 & 6 & 3 & 2 & {$[3,2,2,3]$} & {$[12,3]$} & {$[12]$} \\
\hline $9 a z$ & 96 & 12 & 4 & 1 & {$[2,2,3,2,2,2]$} & {$[24,4]$} & {$[24,2,2]$} \\
\hline $9 b a$ & 24 & 6 & 2 & 1 & {$[2,3,2,2]$} & {$[12,2]$} & {$[12,2]$} \\
\hline $9 b b$ & 144 & 6 & 12 & 2 & {$[2,2,3,3,2,2]$} & {$[12,12]$} & {$[12,2,2]$} \\
\hline $9 b c$ & 12 & 12 & 1 & 1 & {$[2,3,2]$} & [] & {$[12]$} \\
\hline $9 b d$ & 18 & 18 & 1 & 1 & {$[3,3,2]$} & [] & {$[18]$} \\
\hline $9 b e$ & 18 & 18 & 1 & 1 & {$[3,3,2]$} & {[]} & {$[18]$} \\
\hline $9 b f$ & 48 & 6 & 4 & 1 & {$[2,3,2,2,2]$} & {$[12,4]$} & {$[12,2,2]$} \\
\hline $9 b g$ & 48 & 12 & 2 & 1 & {$[2,3,2,2,2]$} & {$[24,2]$} & {$[24,2]$} \\
\hline $9 b h$ & 192 & 6 & 8 & 1 & {$[2,2,3,2,2,2,2]$} & {$[24,8]$} & {$[24,2,2,2]$} \\
\hline $9 b i$ & 384 & 6 & 8 & 1 & {$[2,2,2,3,2,2,2,2]$} & {$[48,8]$} & {$[48,4,2]$} \\
\hline $9 b j$ & 192 & 6 & 4 & 1 & {$[2,2,2,3,2,2,2]$} & {$[48,4]$} & {$[48,2,2]$} \\
\hline $9 b k$ & 144 & 12 & 6 & 2 & {$[2,3,2,2,2,3]$} & {$[24,6]$} & {$[24,2]$} \\
\hline $9 b l$ & 96 & 12 & 4 & 1 & {$[3,2,2,2,2,2]$} & {$[24,4]$} & {$[24,2,2]$} \\
\hline $9 b m$ & 96 & 12 & 4 & 1 & {$[3,2,2,2,2,2]$} & {$[24,4]$} & {$[24,2,2]$} \\
\hline $9 b n$ & 4320 & 6 & 720 & 720 & {$[2,360,2,3]$} & {$[6]$} & [6] \\
\hline $9 b o$ & 24 & 24 & 1 & 1 & {$[2,2,3,2]$} & [] & [24] \\
\hline $9 b p$ & 864 & 6 & 72 & 12 & {$[2,3,2,3,3,2,2,2]$} & {$[12,9,4,2]$} & {$[12]$} \\
\hline $9 b q *$ & 96 & 12 & 4 & 1 & {$[3,2,2,2,2,2]$} & {$[24,4]$} & {$[24,2,2]$} \\
\hline $9 b r$ & 1536 & 6 & 64 & 1 & {$[2,2,3,2,2,2,2,2,2,2]$} & {$[24,64]$} & {$[24,2,4,4,2]$} \\
\hline $9 b s$ & 12 & 12 & 1 & 1 & {$[3,2,2]$} & [] & [12] \\
\hline $9 b t$ & 24 & 24 & 1 & 1 & {$[2,2,3,2]$} & {[]} & {$[24]$} \\
\hline $9 b u$ & 60 & 6 & 5 & 2 & {$[2,3,5,2]$} & {$[12,5]$} & {$[12]$} \\
\hline $9 b v$ & 1152 & 6 & 48 & 6 & {$[2,2,2,3,2,3,2,2,2]$} & {$[24,6,4,2]$} & {$[24,2]$} \\
\hline $9 b w$ & 48 & 6 & 4 & 1 & {$[2,3,2,2,2]$} & {$[12,4]$} & {$[12,2,2]$} \\
\hline $9 b x$ & 96 & 6 & 8 & 1 & {$[2,3,2,2,2,2]$} & {$[12,8]$} & {$[12,2,2,2]$} \\
\hline $9 b y$ & 96 & 12 & 4 & 1 & {$[2,3,2,2,2,2]$} & {$[24,4]$} & {$[24,2,2]$} \\
\hline $9 b z$ & 864 & 6 & 36 & 8 & {$[2,2,2,3,2,2,3,3]$} & {$[24,4,9]$} & {$[24,2,2]$} \\
\hline $9 c a$ & 192 & 12 & 8 & 1 & {$[2,2,2,2,2,3,2]$} & {$[24,8]$} & {$[24,4,2]$} \\
\hline $9 c b$ & 288 & 6 & 24 & 2 & {$[2,2,3,3,2,2,2]$} & {$[12,24]$} & {$[12,2,2,2]$} \\
\hline $9 c c$ & 576 & 6 & 24 & 6 & {$[2,3,2,2,3,2,2,2]$} & {$[24,3,4,2]$} & [24] \\
\hline $9 c d$ & 96 & 12 & 4 & 1 & {$[2,3,2,2,2,2]$} & $[24,4], 2]$ & {$[24,2,2]$} \\
\hline $9 c e$ & 480 & 6 & 20 & 4 & {$[2,2,5,3,2,2,2]$} & {$[24,20]$} & {$[24,2,2]$} \\
\hline $9 c f$ & 36 & 6 & 3 & 2 & {$[3,2,2,3]$} & {$[12,3]$} & {$[12]$} \\
\hline $9 \mathrm{cg}$ & 768 & 6 & 32 & 1 & {$[2,3,2,2,2,2,2,2,2]$} & {$[24,32]$} & {$[24,4,4,2]$} \\
\hline $9 \mathrm{ch}$ & 1152 & 6 & 96 & 6 & {$[2,3,2,2,2,3,2,2,2]$} & {$[12,12,4,2]$} & {$[12,2,2]$} \\
\hline $9 c i$ & 288 & 6 & 12 & 2 & {$[2,2,2,3,2,3,2]$} & {$[24,12]$} & {$[24,2,2]$} \\
\hline $9 c j$ & 384 & 6 & 16 & 1 & {$[2,3,2,2,2,2,2,2]$} & {$[24,16]$} & {$[24,2,2,2,2]$} \\
\hline $9 c k$ & 1152 & 6 & 24 & 2 & {$[2,2,2,3,2,2,3,2,2]$} & {$[48,24]$} & {$[48,2,2,2]$} \\
\hline $9 \mathrm{cl}$ & 120 & 6 & 5 & 4 & {$[2,3,2,2,5]$} & {$[24,5]$} & {$[24]$} \\
\hline $9 \mathrm{~cm}$ & 384 & 12 & 16 & 1 & {$[2,2,3,2,2,2,2,2]$} & {$[24,16]$} & {$[24,4,2,2]$} \\
\hline $9 \mathrm{cn}$ & 1728 & 6 & 72 & 12 & {$[2,3,2,2,3,3,2,2,2]$} & {$[24,9,4,2]$} & {$[24]$} \\
\hline $9 \mathrm{co}$ & 1152 & 6 & 48 & 6 & {$[2,2,3,2,2,3,2,2,2]$} & {$[24,6,4,2]$} & {$[24,2]$} \\
\hline $9 c p$ & 576 & 12 & 24 & 2 & {$[2,2,3,2,2,2,2,3]$} & {$[24,24]$} & {$[24,4,2]$} \\
\hline $9 c q$ & 13824 & 6 & 1152 & 72 & {$[2,3,2,2,3,3,2,2,2,2,2,2]$} & {$[12,2,9,16,4]$} & {$[12,2]$} \\
\hline $9 \mathrm{cr}$ & 576 & 12 & 24 & 2 & {$[2,2,3,3,2,2,2,2]$} & {$[24,24]$} & {$[24,2,2,2]$} \\
\hline $9 c s$ & 2880 & 6 & 240 & 120 & {$[2,3,2,60,2,2]$} & {$[12,2]$} & {$[12,2]$} \\
\hline $9 c t$ & 11520 & 6 & 1920 & 120 & {$[2,60,2,2,2,2,2,3]$} & [6] & [6] \\
\hline $9 c u$ & 11289600 & 48 & 940800 & 235200 & {$[2,2,3,2,2,2,58800,2]$} & {$[12,8]$} & {$[12,2,2,2]$} \\
\hline
\end{tabular}

\section{REFERENCES}

[1] A. Aho, B. W. Kernighan and P. J. Weinberger. The AWK Programming Language. AddisonWesley, Reading, Mass., 1988.

[2] R. D. Baker and G. L. Ebert, Construction of two-dimensional flag-transitive planes. Geom. Dedicata, 27 (1988), 9-14. MR 89f:51017

[3] C. Charnes, Ph.D. thesis, Cambridge University, 1989.

[4] C. Charnes, Quadratic matrices and the translation planes of order $5^{2}$. Coding Theory, Design Theory, Group Theory, Proceedings of the M. Hall Conference (Eds. D.Jungnickel, S.A. Vanstone), J. Wiley and Sons, Inc. New York, 1993, pp.155-161. MR 94h:51016 
[5] C. Charnes, A pair of mutually polar translation planes. Ars. Comb., 37 (1994), 121-128. MR 95d:05028

[6] C. Charnes and U. Dempwolff, Involutory homologies and translation planes of order 49. Abstracts Australian Mathematical Society Annual Conference, University of Wollongong, July 5-9, 1993.

[7] C. Charnes and U. Dempwolff, Spreads ovoids and $S_{5}$, Geom. Dedicata, 56 (1995), 129-143.

[8] J. H. Conway, P. B. Kleidman and R. A. Wilson, New families of ovoids in $\mathrm{O}_{8}^{+}$. Geom. Dedicata, 26 (1988), 157-170.

[9] P. Dembowski, Finite Geometries. Springer-Verlag, Berlin, 1968.

[10] U. Dempwolff, Translation planes of order 27. Designs, Codes and Cryptography, 4 (1994), 105-121.

[11] G. Heimbeck, Translationsebenen der Ordnung 49 mit einer Quaternionengruppe von Dehnungen. Journal of Geometry, 44 (1992), 65-76.

[12] G. Heimbeck, Translationsebenen der Ordnung 49 mit Scherungen. Geom. Dedicata, 27 (1988), 87-100.

[13] U. Dempwolff and A. Reifart, The classification of the translation planes of order 16 I, Geom. Dedicata, 15 (1983), 137-153.

[14] H. Lüneburg, Translation Planes. Springer-Verlag, Berlin, New York, 1980.

[15] R. Mathon and G. Royle, The translation planes of order 49. Designs, Codes and Cryptography, 5 (1995), 57-72.

[16] M. Schönert et al., GAP Groups, Algorithms and Programming 3.3, Lehrstuhl D für Mathematik, RWTH Aachen, 1993.

[17] E. E. Shult, A sporadic ovoid in $\Omega^{+}(8,7)$. Algebras, Groups and Geometries, 2 (1985), 495513.

Department of Computer Science University of Wollongong, FB Mathematik UniVERSITÄT KAISERSLAUTERN

E-mail address: charnes@cs.uow.edu.au

E-mail address: dempwolff@mathematik.uni-kl.de 